\title{
ThE PRODUCTION OF CONSCIOUSNESS OUT OF STATES OF CONSCIOUSNESS
}

\author{
Todd Murphy* \\ Neuroscience Research Group, Laurentian University, Sudbury, Canada \\ Received March 10, 2009; accepted April 2, 2009
}

\begin{abstract}
It has been found that there are five million magnetite crystals per gram in the human brain. Interestingly, the meninges, has twenty times that number. These 'biomagnetite' crystals demonstrate two interesting features. The first is that their shapes do not occur in nature, suggesting that they were formed in the tissue, rather than being absorbed from outside. The other is that these crystals appear to be oriented so as to maximize their magnetic moment, which tends to give groups of these crystals the capacity to act as a system. We hypothesize that the entire set of modalities, cognitive and sensory, may be heuristically compared to a sound mixing board. In this metaphor, all the various modalities are represented as vertical rheostats with enhanced functioning increasing towards the top, and suppressed function increasing towards the bottom. Further, the act of becoming conscious of phenomena in any given modality involves the adjustment of that modality's 'rheostat'.
\end{abstract}

Key words: Magnetoencephalography; Consciousness; Subjective Awareness

\section{NEUROMAGNETIC SIGNALS AS THE BASIS FOR STATES OF CONSCIOUSNESS}

In what could turn out to be one of the most important discoveries in cognitive studies of our decade, it has been found that there are five million magnetite crystals per gram in the human brain (Kirshivink, KobayashiKirshivink, \& Woodford, 1992). Interestingly, the meninges, (the membrane that envelops the brain), has twenty times that number. These 'biomagnetite' crystals demonstrate two interesting features. The first is that their shapes do not occur in nature, suggesting that they were formed in the tissue, rather than being absorbed from outside. The other is that these crystals appear to be oriented so as to maximize their magnetic moment, which tends to give groups of these crystals the capacity to act as a system. The brain has also been found to emit very low intensity magnetic fields, a phenomenon that forms the basis of a whole diagnostic field, Magnetoencephalography (Stefan et al., 1994).

Unfortunately for the present discussion, there is no way to 'read' any signals that might be carried by the brains magnetic emissions at present. We expect that subtle enough means of detecting such signals will eventually appear, as there is compelling evidence that they do exist, and constitute a means whereby commu- nication happens between various parts of the brain. This system, we speculate, is what makes the selection of which neural areas to recruit, so that States (of consciousness) can elicit the appropriate phenomenological, behavioral, and affective responses.

While there have been many studies that have examined the effects of magnetic fields on human consciousness, none have yielded findings more germane to understanding the role of neuromagnetic signaling than the work of the Laurentian University Behavioral Neurosciences group. They have pursued a course of experiments that rely on stimulating the brain, especially the temporal lobes, with complex low intensity magnetic signals (Ruttan, Persinger, \& Koren, 1990). It turns out that different signals produce different phenomena.

One example of such phenomenon is vestibular sensation, in which one's normal sense of balance is replaced by illusions of motion similar to the feelings of levitation reported in spiritual literature as well as the sensation of vertigo. Transient 'visions', whose content includes motifs that also appear in near-death experiences and alien abduction scenarios have also appeared (Persinger, 1993). Positive affectual parasthesias (electriclike buzzes in the body) have occurred. Another experience that has been elicited neuromagnetically is bursts

\footnotetext{
*Correspondence to: Todd Murphy, e-mail:brainsci@jps.net
} 
of emotion, most commonly fear and joy. Although the content of these experiences can be quite striking, the way they present themselves is much more ordinary. It approximates the 'twilight state' between waking and sleep called hypnogogia. This can produce brief, fleeting visions, feelings that the bed is moving, rocking, floating or sinking. Electric-buzz like somatic sensations and hearing an inner voice call one's name can also occur in hypnogogia. The range of experiences it can produce is quite broad. If all signals produced the same phenomena, then it would be difficult to conclude that these magnetic signals approximate the postulated endogenous neuromagnetic signals that create alterations in State. In fact, the former produce a wide variety of phenomena. One such signal makes some women apprehensive, another doesn't (Richards, Persinger, \& Koren, 1992). One signal creates such strong vestibular sensations that one can't stand up. Another doesn't.

The temporal lobes are the parts of the brain that mediate states of consciousness. EEG readouts from the temporal lobes are markedly different when a person is asleep, having a hallucinogenic seizure, or on LSD. Seizural disorders confined to the temporal lobes (complex partial seizures) have been characterized as impairments of consciousness (Feldman, 1983). There was also a study done in which monkeys were given LSD after having various parts of their brains removed (Baldwin, 1970). The monkeys continued to 'trip' no matter what part or parts of their brains were missing until both temporal lobes were taken out. In these cases, the substance did not seem to affect the monkeys at all. The conclusion seems unavoidable. In addition to all their other functions (aspects of memory, language, music, etc.), the temporal lobes mediate states of consciousness.

If exposing the temporal lobes to magnetic signals can induce alterations in States, then it seems reasonable to suppose that States find part of their neural basis in our postulated neuromagnetic signals, arising out of the temporal lobes.

Hallucinations are known to be the phenomenological correlates of altered States. Alterations in state of consciousness lead, following input, and phenomena, whether hallucinatory or not, follow in response. We can offer two reasons for drawing this conclusion.

The first is one of the results obtained by a study of hallucinations caused by electrical stimulation deep in the brain (Horowitz \& Adams, 1970). In this study, the content of the hallucinations was found to be related to the circumstances in which they occurred, so that the same stimulations could produce different hallucinations. The conclusion was that the stimulation induced altered states, and the states facilitated the hallucinations.

The second has to do with the relative speeds of the operant neural processes.
Neurochemical response times are limited by the time required for their transmission across the synaptic gap, 0.5 to $2 \mathrm{msec}$ (Stevens, 1979). By comparison, the propagation of action potentials is much faster. For example, an action potential can travel a full centimeter (a couple of orders of magnitude larger than a synaptic gap) in about $1.3 \mathrm{msec}$. The brain's electrical responses, therefore, happen orders of magnitude more quickly than do it's chemical ones (Kalat, 1981).

Magnetic signals are propagated with much greater speeds than those of action potentials moving through neurons. Contemporary physics requires that magnetic signals be propagated at a significant fraction of the velocity of light, so that the entire brain could be exposed to a neuromagnetic signal in vanishingly small amounts of time.

It seems possible that neuromagnetic signals arise from structures which mediate our various sensory and cognitive modalities. These signals then recruit those functions (primarily in the limbic system) that adjust the changes in state. These temporal lobe signals, we speculate, then initiate signals to structures that mediate modalities that are enhanced or suppressed as the state changes.

\section{CONSCIOUSNESS AS A FEEDBACK INTER- FACE OF SENSORY AND COGNITIVE MODAL- ITIES}

The problem of defining the phrase 'state of consciousnesses' has plagued the field of cognitive studies for some time. Without going into the history of studies in the area, we would like to outline an hypothesis concerning states of consciousness in which the management of states gives rise to the phenomenon of consciousness.

There are theories that suggest that cognitive modalities (such as memory, affect, ideation and attention) may be seen as analogs to sensory modalities.

We hypothesize that the entire set of modalities, cognitive and sensory, may be heuristically compared to a sound mixing board. In this metaphor, all the various modalities are represented as vertical rheostats with enhanced functioning increasing towards the top, and suppressed function increasing towards the bottom. Further, the act of becoming conscious of phenomena in any given modality involves the adjustment of that modality's 'rheostat'.

Sensory input from any modality can alter one's state. The sight of a sexy person, the smell of fire, the unexpected sensation of movement against one's skin (there's a bug on me!), a sudden bitter taste experienced while eating ice cream, or the sound of one's child screaming in pain; all of these phenomena can induce alterations in State. Although the phrase 'altered states' has come to be associated with dramatic, otherworldly experiences, alterations in state, as we will be using the phrase, refers 
primarily to those alterations that take us from one normal state to another. Alterations in state can create changes within the various sensory and cognitive modalities. An increase in arousal following the sight of a predator will typically suppress the sense of smell (very few are able to stop and 'smell the roses' while a jaguar is chasing them), suppress introspection (nobody wants to know 'who am I really?' while an anaconda is wrapping itself around them), suppress sexual arousal, and alter vision so that the center of the visual field is better attended then one's peripheral vision allowing one to see the predator's movement better. The sight of a predator will also introduce a host of other changes, all of which reflect the State.

In the Hindu epic, the Mahabharata, there is a dialog between the legendary warrior, Arjuna, and his archery teacher. Arjuna was told by his teacher to train his bow on a straw bird used as a target. Arjuna was asked to describe the bird. He answered 'I can't'. 'Why not?', asked his teacher. 'I can only see its eye', he answered. 'Release your arrow', commanded the teacher. Arjuna did, and hit the target in the eye. 'I'll make you the finest archer in the world', said his teacher.

In this story, attention to peripheral vision had ceased so completely that only the very center of his visual field received any. Our model of states would be constrained to interpret Arjuna's (mythical) feat as a behavior specific to a state. The unique combination of sensory enhancement, heightened attention, and sufficient suppression of emotion, ideation, and introspection that support such an act suggests specific settings for our metaphorical rheostats.

Changes in state make changes in sensory and cognitive modalities, and they in turn, trigger changes in state. We can reasonably conclude that there is a feedback mechanism whereby each modality is connected to the others.

States also create tendencies to behave in specific ways in specific circumstances, maximizing the adaptivity of behavior in those circumstances; behavior that tends to meet our needs and respond to threats to our ability to meet those needs.

Each circumstance adjusts each 'modalities' setting, tending to maximize that modality's contribution to adaptive behavior in that circumstance. The mechanism may function by using both learned and inherited default settings for each circumstance and then repeating those settings in similar circumstances later on. Sadly, this often makes states maladaptive. A habituated alteration in State, in response to threats from an abusive parent, for example, can make for self-defeating responses to stress in other circumstances, where theses same responses are no longer advantageous (Perry et al., 1995).

Because different States are going to be dominated by specific combinations of modalities, it stands to reason that a possible strategy for aligning the rheostats (mak- ing alterations in state) is to move them in tandem, so that after a person associates the sound of a scream to the concept of a threat, that sound, with it's unique auditory signature, will cause all the affected modalities (most likely most of them in most cases) to take the positions they had at the time the association was made. When we say changing states, we are referring to much more than the dramatic states created by LSD, isolation tanks, REM sleep, etc. We are also including normal states of consciousness, which we can imagine as kindled 'default settings' of our various modalities. When any one of these settings returns to one of its default settings, it will, we conjecture, tend to entrain all the other modalities to the settings they habitually take in that state.

To accomplish this, we must suggest that each modality is connected to every other one. A sight, a smell, a sound, or a tactile feeling can all inspire fear. Fear can motivate ideation. Ideation can inspire arousal. Changes in affect can initiate alterations in introspection. Introspection alters affect. State specific settings of individual modalities could initiate settings for other modalities.

Our main hypothesis here is that all these intermodal connections, operating as a single system, has a single phenomenological correlate.

\section{THE PHENOMENA OF SUBJECTIVE AWARE- NESS}

We proposed in our first section that the alteration of consciousness involves having a modality receive input that triggers a change in State. The structure associated with that modality then broadcasts a neuromagnetic signal to the temporal lobes, which then produces signals that then recruits various structures throughout the brain. Specifically, those structures whose associated modalities' values must be changed in order to accomplish the appropriate alteration in state. In the second section, we found the possibility that states are settings for the variable aspects of cognitive and sensory modalities. We also offered the suggestion that consciousness is the phenomenological correlate of the feedback between the management of states on the one hand, and the various cognitive and sensory modalities, on the other. If all of these conclusions were to stand up to testing, we could conclude that the content of the brain's hypothesized endogenous magnetic signals might consist of a set of values for adjusting each sensory and cognitive rheostat. We might also conclude that neuromagnetic signaling is the context in which consciousness occurs.

The specific mechanism whereby subjectivity is generated is out of the reach of this work. Nevertheless, we can say that the fact that multiple modalities are experienced simultaneously, together with our model's implication that they are 'reset,' all at once, with each alteration in state suggests that our postulated neuromagnet- 
ic signals may come in pairs, with the two signals running slightly out of phase with one another. In this way, neuromagnatic signals, like the two laser beams used to produce a hologram, might be able to store information in a similar way, as has already been explored by Karl Pribram. The speeds at which neuromagnetic signals are propagated, together with their capacity to recruit/alter multiple modalities suggests that the underlying mechanism has been selected to make instant choices on which specific portions to recruit in order to facilitate the behaviors acted out of the State, and to do so quickly.

In this way, the onset time for the initiation of States is kept to a minimum, and with it, the times needed to make the initial, cognitive response to stimuli. When it comes to response to threats, or sighting prey, the evolutionary advantages are obvious.

\section{REFERENCES}

Baldwin, M., (1970). Neurologic Syndromes and Hallucinations. In: Origin and Mechanisms of Hallucinations. Keup, Wolfram, (Ed.), New York: Plenum Press.

Feldman, R.G. (1983). Complex Partial Siezures (Psychomotor or Temporal Lobe Siezures). In: Epilepsy-Diagnosis and Management. Eds: Brown, Thomas R., \& Feldman, Robert G., Little, Brown \& Co.: Boston.

Horowitz, M.J. \& Adams, J.E. (1970). Hallucinations on Brain Stimulation: Evidence for the Revision of the Penfield Hypothesis. In: Origin and Mechanism of Hallucinations : Keup, (Ed.), New York: Plenum Press.

Kalat, J.W. (1981). Biological Psychology (2nd Ed.), 46, Belmont: Wadsworth Publishing Co.

Kirshivink, J.L., Kobayashi-Kirshivink, A. \& Woodford, B.J. (1992). Magnetite Biomineralization in the Human Brain. Proceedings of the National Academy of Science, 89, 76837687.

Perry, B.D., Pollard, R.A., Blakley, T.L., Baker, W.L., \& Vigilante, D. (1995). Childhood Trauma, The Neurobiology of Adaptation, and the 'use dependent' Development of the Brain: How 'States' become 'traits.'. Infant Mental Health Journal, 16, 271-291

Persinger, M.A., (1993). Near-Death Experiences: Determining the Neuroanatomical Pathways by Experiential Patterns and Simulation In Experimental Settings, In: Healing: Beyond Suffering and death. Quebec: Publications MNH.

Richards, P.M., Persinger, M.A. \& Koren, S. (1992). Experimental Stimulation by Burst-Firing Weak Magnetic Fields over the Right Temporal Lobe May Facilitate Apprehension in Women. Perceptual and Motor Skills, 75, 667-670.

Ruttan, L.A., Persinger, M.A., \& Koren, S. (1990). Enhancement of Temporal Lobe-Related Experiences During Brief Exposures to Milligaus Intensity Extremely Low Intensity Magnetc Fields. Journal of Bioelectricity 9, 33-54.

Stefan, H., Abraham-Fuchs, K., Shnieder, S., Gebhardt, M. Neubauer, U. Hummel, C., Huk, W.J., \& Thierauf, P. (1994). Magnetic Source Localization and Morphological Changes in Temporal Lobe Epilepsy: Comparison of MEG/EEG, EcoG and Volumatric MRI in Pesurgical Evaluation of Operated Patients. Neurologia Scandinavica. Suppl., 152, 83-8
Stevens, C.F. (1979). The Neuron. In: The Brain W.H. New York: Freeman and Co. 